\title{
¿Las cosas claras? Aspiraciones de futuro y proyecto educativo laboral de jóvenes estudiantes secundarios*
}

\author{
Getting It Straight? Aspirations for the future and educational employment projects for \\ high school youths
As coisas claras? Aspirações para o futuro e projeto educativo trabalhista de jovens estudantes secundários

\author{
Leandro Sepúlveda V., ${ }^{a}$ María José Valdebenito I. ${ }^{a}$ \\ ${ }^{a}$ Centro de Investigación y Desarrollo de la Educación CIDE, Universidad Alberto Hurtado. Telf.: \\ 2-28897100. Correo electrónico: 1sepulve@cide.cl mvaldebe@cide.cl
}

\begin{abstract}
RESUMEN
Este artículo analiza las aspiraciones y expectativas de futuro que construyen estudiantes del último nivel de enseñanza secundaria en una muestra representativa de centros educacionales de la ciudad de Santiago. El estudio demuestra que, independientemente del nivel socio-económico y modalidad de estudios cursados, la gran mayoría de los jóvenes estudiantes aspiran a ingresar a la educación superior y obtener un título profesional. Sin embargo, existen diferencias importantes en la proyección del cumplimiento de los objetivos personales, observándose una mayor apertura a combinar períodos de estudio y trabajo entre los jóvenes de nivel socio-económico bajo y medio-bajo y la postergación del ingreso inmediato a la educación superior para un segmento importante de estos últimos. Se revisan diversas racionalidades presentes en el discurso de los estudiantes, las que darían cuenta de un ajuste de las aspiraciones de futuro, y la elección de carreras y centros de estudios funcionales a su origen social.

Palabras clave: aspiraciones; transición educación-trabajo; estudiantes secundarios; sistema educativo.
\end{abstract}

\begin{abstract}
This article analyzes the aspirations and expectations for the future of senior high school students through a representative sample of educational centers in the city of Santiago. The study shows that, independently of socioeconomic level and the modality of the courses taken, the great majority of students hopes to get into an institution of higher education and obtain a professional degree. However, there are important differences in the perception as to how to achieve these goals, where a large number of young people from low and low-middle income classes are more open to combining work and studies and end up postponing their higher educational studies. Diverse rationalities expressed by students are reviewed, which would explain their expectations for the future and their choice of career and technical studies according to their social status.
\end{abstract}

Key words: aspirations, education-work transitions; high school students; education system.

\section{RESUMO}

Analisam-se as expectativas e aspirações para o futuro construídas por estudantes do último ano do Ensino Médio em uma mostra representativa de centros educacionais da cidade de Santiago. O estudo demonstra que, independente do nível socioeconômico e da modalidade de estudos cursados, a maioria dos jovens estudantes aspira ao ingresso na Educação Superior e à obtenção de um título profissional. No entanto, há diferenças importantes na projeção da concretização dos objetivos pessoais, observando-se maior abertura na combinação de períodos de estudo e trabalho entre os jovens de nível socioeconômico baixo e médio-baixo e o adiamento do ingresso imediato na Educação Superior para

Este artículo se ha desarrollado en el marco del estudio "Expectativas, proyectos educativo-laborales y trayectorias post-egreso de jóvenes estudiantes secundarios: un estudio en la Región Metropolitana". Proyecto FONDECYT 1110544. 
um segmento importante deste. Revisam-se diversas racionalidades presentes no discurso dos estudantes, aquelas que sustentariam um ajuste das aspirações para o futuro e a eleição de carreiras e centros de estudos funcionais consoantes à origem social.

Palavras chave: aspiraç̃es, transição educação-trabalho, estudantes do ensino médio, sistema educativo.

\section{INTRODUCCIÓN}

La ampliación sostenida de la matrícula de educación superior es uno de los rasgos más relevantes de las transformaciones ocurridas en la sociedad chilena en los últimos años, reflejando cambios sustanciales en las aspiraciones de futuro y proyectos educativolaborales de las nuevas generaciones de jóvenes y sus familias. En efecto, mientras que en el año 1990 la matrícula bruta de estudiantes en el sistema alcanzaba al 16,9\% de los jóvenes entre 18 y 24 años, en el año 2011 esta se empinaba al $48 \%$ del total, representando una cifra cercana al millón de personas de este tramo de edad. Este incremento ha sido liderado por la oferta del sector privado de la educación superior que concentra más del 75\% del total de la matrícula. El año 2012 el 26,7\% de los estudiantes concurrían a universidades asociadas al Consejo de Rectores de las Universidades de Chile, CRUCh; el $33,3 \%$ correspondía a estudiantes de universidades privadas no integradas en esta instancia; un $27,3 \%$ a Institutos Profesionales y un $12,7 \%$ a Centros de Formación Técnica (CFT). ${ }^{1}$

Entre las principales características que dan cuenta de este crecimiento, es importante destacar dos hechos relevantes:

(a) La incorporación de jóvenes cuyos padres no poseen estudios de nivel superior, constituyendo un grupo más que significativo dentro del total de estudiantes del sistema. Hacia mediados de la década pasada, se estimaba que el $70 \%$ de la matrícula correspondía a estudiantes de primera generación en la educación superior, esto es, el primer integrante de una familia que ingresaba a algunas de las alternativas de la oferta educativa de este nivel. Estudios recientes señalan que cerca del $80 \%$ de los actuales estudiantes del sistema, con el ingreso a una carrera de educación superior, ha logrado superar el nivel educacional de sus padres, evidenciando un cambio generacional de gran magnitud (Castillo y Cabezas, 2010).

(b) Muy vinculado con lo anterior, en las últimas décadas destaca el aumento del porcentaje de jóvenes de los estratos más pobres de la sociedad en el sistema de educación superior. De acuerdo a los datos de la Encuesta CASEN, entre 1990 y el año 2009, la participación de los dos quintiles más pobres de la población ha aumentado en más tres veces, pasando de una representación de un 3,7\% a un $16,9 \%$ en el primer quintil y de un $6,6 \%$ a un $21,4 \%$ en el segundo. Aunque todavía se observan evidentes diferencias por nivel socio-económico, ${ }^{2}$ los análisis recientes señalan una tendencia a la saturación de la representación en el sistema de los estratos más acomodados, existiendo la posibilidad cierta de que en el futuro la expansión estará concentrada en los estratos medios y bajos de la población (Orellana, 2011).

\footnotetext{
Consejo Nacional de Educación, datos sistema de educación superior 2011-2012; http://www.cned.cl
}

2 La diferencia en la participación del primer quintil respecto al quinto quintil más rico en la educación superior es de 40 puntos porcentuales. 
Desde una perspectiva general, el sistema de educación superior ha dejado de ser una instancia destinada exclusivamente para las elites, presentando mayores niveles de complejidad y nuevas exigencias para su desarrollo. Algunas líneas de investigación recientes intentan abordar estos desafíos que incluyen, entre otros, los requerimientos de ajuste organizacional necesarios en el sistema de educación superior en este nuevo escenario (Gaete y Morales, 2011), la reflexión sobre el tipo de estrategias de retención de estudiantes en el sistema, muy particularmente los de condición vulnerable (Donoso et al., 2010) o la necesidad de una mayor transparencia de información acerca de la rentabilidad e inserción laboral que ofrece la oferta formativa actualmente vigente (Meller, 2010).

Estos y otros esfuerzos investigativos centralizan su preocupación en el análisis institucional y las propuestas de cambio necesarios para la asunción de sus transformaciones recientes. Con todo, un ámbito que ha merecido menor atención y que, sin embargo, resulta muy relevante en el análisis de las transformaciones del sistema de educación superior y sus efectos en la organización social, remite a las aspiraciones y proyectos educativos que construyen los jóvenes en la etapa previa a su egreso de la enseñanza secundaria y el nivel de correspondencia de tales aspiraciones con la realidad posterior. La consideración de los procesos vivenciados por los sujetos y las estrategias que éstos desarrollan para hacer frente a sus condiciones de vida es un tema débilmente abordado existiendo, por lo general, una distancia analítica con esta perspectiva en la investigación educativa realizada en Chile en los últimos años. ¿Qué tipo de formación y qué centro de estudios privilegian los jóvenes en la definición de sus proyectos personales? ¿Qué influencia tienen el tipo de establecimiento educacional al que concurren en la definición de sus aspiraciones? ¿Qué certezas tienen los estudiantes de alcanzar sus objetivos y qué factores pueden incidir en el logro o fracaso de sus planes? ¿Cómo se articula la educación y el trabajo en esos planes de futuro? Son algunas preguntas relevantes que pueden ser levantadas desde este ámbito de la investigación.

Estudios recientes en sociología de la juventud remarcan la importancia de considerar la subjetividad y las estrategias de acción de los sujetos en el análisis de los cambios ocurridos en el sistema educativo en los últimos años. Un número considerable de investigaciones sobre la construcción de aspiraciones en los estudiantes y los procesos de elección de carrera educativa y/o laboral, dan cuenta de los rasgos de incertidumbre creciente que pueden experimentar los jóvenes en esta etapa de decisiones. De igual manera, la consideración acerca del grado de libertad o condicionamiento estructural en las opciones de futuro de los estudiantes, ha llevado a profundizar en la discusión sobre el efecto de la masificación de la educación superior y su eventual aporte a una mayor democratización e igualdad social (Lehmann, 2004; Grytnes, 2011).

Situados en esta perspectiva de investigación, en este artículo se recogen antecedentes de un estudio en desarrollo sobre la trayectoria educativa laboral de jóvenes estudiantes chilenos en su último nivel de enseñanza media. El material que aquí se revisa proviene de la primera fase de levantamiento de información empírica de este estudio, e intenta dar cuenta del tipo de aspiraciones que construyen estudiantes de establecimientos educacionales de diverso nivel socio-económico, y las racionalidades o lógicas sociales que enmarcan tales decisiones. 


\section{MARCO DE ANÁLISIS}

En el ámbito de la investigación educativa existe un amplio consenso para reconocer que las aspiraciones que construyen los sujetos constituyen uno de los más importantes predictores de sus resultados educacionales y laborales futuros. A partir de esta constatación, una parte relevante de la investigación desarrollada en las últimas décadas se ha focalizado en el interés por identificar algunas variables que pueden incidir en las diferencias observadas en el tipo de aspiraciones predominantes a nivel social; entre otras, el nivel educacional de los padres, el género, el contexto institucional, el compromiso académico de la familia o la propia experiencia escolar de los estudiantes (Berzin, 2010). Aunque en muchos de estos estudios es posible establecer una asociación significativa de una o más variables con las aspiraciones y logros educacionales, por lo general, su tratamiento se ha quedado en un nivel descriptivo, sin profundizar en cómo y por qué estos factores influyen de manera consistente en tales orientaciones (Jones y Schneider, 2009).

Una perspectiva que profundiza teóricamente en el tratamiento de esta temática proviene de la influencia del trabajo de Bourdieu y su concepto de capital cultural como forma de transmisión y reproducción de las clases sociales. McDonough (1997) utiliza este enfoque para demostrar que las aspiraciones y el tipo de elección de educación superior que realizan los estudiantes de nivel secundario, son condicionadas por el contexto social, cultural y organizacional del cual provienen, jugando un rol fundamental el tipo de establecimiento educacional donde los jóvenes han cursado su enseñanza secundaria.

En este marco, las aspiraciones individuales responden a percepciones compartidas por los miembros de un grupo o clase social y que orienta el tipo de elección que les resulta posible. Son evaluaciones subjetivas acerca de las posibilidades de movilidad social y de construcción de una trayectoria personal, pero representan, al mismo tiempo, las probabilidades objetivas de futuro de quienes las construyen. No son análisis racionales, pero constituyen la forma en que los jóvenes de diversas clases sociales construyen elecciones razonables en función del capital cultural que disponen. El estudio de McDonough aporta dos elementos conceptuales relevantes, (a) por una parte, extiende el concepto de habitus y presenta evidencia de que este no solo existe en las familias y comunidades, sino que también en el propio espacio escolar, operando como un habitus organizacional que es utilizado e influye en la construcción de las decisiones individuales, y (b) esta autora utiliza el concepto de racionalidad limitada para indicar que las decisiones personales y el tipo de elección al que llegan los jóvenes, están acotadas de acuerdo al grado de constreñimiento social que evidencia su propio capital cultural.

La investigación reciente sobre este tema profundiza en dos aspectos íntimamente relacionados y que hacen referencia a las transformaciones del contexto; por un parte, el incremento de las aspiraciones educativas de los jóvenes y sus familias y, por otro, el tipo de racionalidad emergente en un marco de una oferta educativa en expansión.

Schneider y Stevenson (1999) analizan los cambios acaecidos en la nueva generación de adolescentes y jóvenes en comparación a la de sus padres en los Estados Unidos. En un estudio representativo a nivel nacional, los autores destacan la explosión de aspiraciones fundadas en una mayor permanencia en el sistema escolar y en los proyectos que allí se diseñan. Los autores advierten sobre la existencia de ambiciones desalineadas (subestimación de la educación necesaria para alcanzar una meta), las que pueden incidir 
fuertemente en el tipo de estrategias de incorporación a la vida laboral una vez terminado el período de educación obligatoria.

En América Latina, estudios recientes también abordan la creciente masificación del sistema escolar y la expansión de las aspiraciones en estudiantes de todos los sectores sociales. Meo y Dabenigno (2010) en un estudio focalizado en jóvenes populares en la ciudad de Buenos Aires destacan que, pese a la persistencia de un sistema educativo altamente segmentado y con tasas significativas de fracaso escolar, la mayoría de los estudiantes consideran a la escuela secundaria como un medio para la integración social y mejoramiento de las condiciones de vida futura. En sus discursos, los jóvenes aspiran a cursar carreras post-secundarias en el futuro inmediato, postergando la inserción en el mundo laboral. Siguiendo los planteamientos de Dubet y Martuccelli (1998), en este estudio se reconoce una orientación instrumental de los jóvenes hacia el sistema escolar y una tendencia significativa en el discurso de estos a reconocerse como un sujeto activo y en condiciones de darle sentido a su experiencia.

La apertura al análisis de las estrategias activas de desarrollo personal y construcción de proyectos de vida, sin obviar la situación de condicionamiento estructural que experimentan los sujetos, es una perspectiva emergente y que ha alimentado nuevos estudios en el marco de la incorporación de jóvenes de nivel socio-económico bajo a la educación superior. Lehmann (2009) observa que en un escenario de masificación del sistema universitario, los jóvenes de clase trabajadora tienen acceso a nuevas experiencias, relaciones e información que inciden en una dislocación del habitus que estos portan, permitiendo el desarrollo de estrategias, algunas exitosas y otras no, para la integración y permanencia en el sistema de educación superior.

En una perspectiva similar, Sellar et al. (2011) analizando el incremento de las aspiraciones por educación superior en la sociedad australiana, señalan que las características de la modernización y globalización del mundo contemporáneo han incidido en el desarrollo de la imaginación y el crecimiento de las aspiraciones de los sujetos y, muy particularmente, los de clases trabajadoras. Para estos autores, la imaginación ha adquirido un nuevo poder en la vida social, toda vez que, mucho más que antes, las personas consideran un amplio rango de alternativas posibles de vida. Por cierto, esto no significa que la capacidad para cumplir tales aspiraciones sean igualitarias o estén mejor distribuidas, pero el campo del quehacer cultural en una perspectiva dinámica, ampliando su función meramente reproductiva, tal como ha sido abordado en análisis precedentes.

Estos autores destacan el aporte de Appadurai para el desarrollo de una perspectiva de análisis más complejo. Para este autor, la capacidad de aspirar ha sido mayormente trabajada desde la ciencia económica, reduciendo su uso a la agencia individual y referida a deseos, preferencias y cálculos centrados, fundamentalmente, en el dominio del mercado. Appadurai señala que esta perspectiva limita una concepción más amplia del concepto de aspiraciones, el que debe ser abordado como un concepto propiamente cultural, que incluye no solo mundos imaginados por grupos sociales, sino que también normas de acción y un sentido común que orienta el quehacer de los sujetos. De este modo, la cultura de la aspiración es una "capacidad social de navegación" que provee un mapa de normas que seguir y que orienta la realización de acciones colectivas. Las aspiraciones se distinguen de las expectativas, que remiten mayormente a los juicios de lo que probablemente ocurra dada la situación actual; las aspiraciones, en cambio, son afirmaciones de voluntad, definiciones de los deseable y, por lo mismo, un marco de 
orientación conductual: lo que se está dispuesto a hacer para que eso ocurra (Appadurai, 2004).

La consideración del concepto de aspiraciones desde este punto de vista señala la relevancia de analizar la subjetividad de los sujetos (sus proyectos personales, ambiciones o deseos) y su rol activo en la definición de itinerarios y trayectorias distintivas, equilibrando una mirada que, hasta ahora, ha estado mayormente centrada en la dimensión institucional del sistema educativo.

\section{METODOLOGÍA}

Los antecedentes que se presentan en este artículo provienen del levantamiento de información empírica correspondiente a la primera fase del estudio "Expectativas, proyectos educativo-laborales y trayectorias post-egreso de jóvenes estudiantes secundarios: Un estudio en la Región Metropolitana", que se realiza con el apoyo de FONDECYT. ${ }^{3}$ En esta fase del estudio se implementó una encuesta dirigida a una muestra de estudiantes del último año de enseñanza media pertenecientes a diversos tipos de establecimientos educacionales de la Región Metropolitana. En términos operativos, el trabajo de campo para esta primera parte del estudio se efectuó durante los meses de mayo y noviembre del año 2011, y contempló la aplicación de un instrumento estructurado a 1888 jóvenes estudiantes de $4^{\circ}$ año medio en 69 establecimientos educativos. ${ }^{4}$

En la construcción de esta muestra se consideró la dependencia administrativa de los centros educacionales seleccionados y la modalidad de estudios de los jóvenes encuestados (modalidad científico-humanista, EMCH y técnico profesional, EMTP). La composición de la muestra es de $34,3 \%$ de estudiantes que cursaban sus estudios en establecimientos de dependencia municipal, un 43,8\% correspondió a jóvenes de establecimientos de carácter particular subvencionado, un 14,9\% a estudiantes de centros educativos particulares pagados y un $7,2 \%$ de la muestra fueron estudiantes que cursaban sus estudios en establecimientos de administración delegada. Del total de encuestados, un 38,3\% estudió la modalidad científico humanista, un 53,5\% alguna especialidad técnico profesional, mientras que un $8,1 \%$ correspondió a estudiantes jóvenes (menos de 21 años de edad) que cursaban su último año de enseñanza media bajo alguna modalidad de educación de adultos.

Con el objetivo de enriquecer el análisis a partir de la realidad de los propios estudiantes, el procesamiento de los resultados incluyó también la consideración del nivel socioeconómico de los establecimientos participantes del estudio. Ésta fue construida en base a la caracterización que establece el Ministerio de Educación a través de la prueba SIMCE y que diferencia a los centros educacionales en relación a la situación

3 Este estudio, de carácter longitudinal, tiene como objetivo analizar los modelos de trayectoria educativo/laboral que experimentan los jóvenes una vez egresados de la enseñanza media. La primera fase de levantamiento de información que sirve de sustento a este artículo se realizó durante el año 2011, seguido de dos etapas sucesivas de indagación a la muestra inicial en los años 2012 y 2013.

4 Los criterios de selección de la muestra consideraron como unidad primaria de muestreo los establecimientos educativos. Estos fueron seleccionados de manera aleatoria según su dependencia administrativa y modalidad de estudio. Adicionalmente, en cada una de las unidades se seleccionó a uno o dos grupos curso del nivel de $4^{\circ}$ medio para la aplicación del instrumento. El cuestionario, que incluyó preguntas destinadas a la caracterización general de los estudiantes, su experiencia educativa, intereses personales y consumo cultural, aspiraciones y proyectos de futuro, entre otras dimensiones, fue de carácter auto-aplicado y con la presencia de un encuestador en la sala de clases quien supervisó el desarrollo del proceso. 
socio-económica promedio de las familias asociadas a cada establecimiento. A partir de este ejercicio, la muestra de este estudio se compone de un $8,8 \%$ de jóvenes pertenecientes al nivel socio-económico bajo, un 34,5\% en el nivel socio-económico medio-bajo, un $19,8 \%$ en el nivel medio, un 19,7\% en el nivel socio-económico medio alto y un $11,5 \%$ de estudiantes que cursaban sus estudios en establecimientos de nivel socio-económico alto.

Finalmente, de manera complementaria a la aplicación del instrumento estructurado, en el marco de este estudio se desarrollaron ocho grupos de discusión con estudiantes de diversos tipos de establecimientos y modalidad de enseñanza. En estas actividades, se profundizó en aspectos cualitativos de las expectativas, intereses y aspiraciones de los jóvenes participantes, siendo el foco principal de la reflexión colectiva, el proyecto de futuro una vez egresados de la enseñanza media. En la presentación de resultados que sigue, se incluyen algunas citas textuales de estudiantes participantes de esta actividad, como una manera de refrendar los principales hallazgos del estudio.

\section{PRINCIPALES RESULTADOS}

Para el análisis de las aspiraciones y los proyectos educativo-laborales de los jóvenes estudiantes, en este artículo se revisan tres dimensiones principales: (a) el proyecto de futuro y la vinculación entre educación y trabajo en sus proyecciones personales; (b) la auto-percepción del manejo de competencias y el pronóstico de resultados académicos al finalizar la enseñanza secundaria; y (c) los criterios u orientaciones en la elección de estudios superiores en estos jóvenes. Las siguientes páginas están destinadas al desarrollo de estos temas a partir de la evidencia recogida en esta fase del estudio.

\subsection{ASPIRACIONES DE FUTURO: EDUCACIÓN Y TRABAJO EN EL PROYECTO DE VIDA DE LOS JÓVENES ESTUDIANTES}

El proyecto personal que construyen los jóvenes estudiantes, refleja el interés generalizado por ingresar a un centro de educación superior previo al inicio de la vida laboral, ratificando el carácter transversal de la aspiración a una calificación de carácter profesional post-secundaria. Esta tendencia no encuentra diferencias de género y solo se presentan distinciones marginales por nivel socio-económico y modalidad e estudios cursados en la enseñanza media.

En efecto, sólo un porcentaje cercano al $8 \%$ de los encuestados en esta indagación señala su disposición a incorporarse al mundo del trabajo de manera inmediata, sin que exista en su horizonte de futuro el interés por continuar estudios post-secundarios. El mayor porcentaje de quienes se encuentran en esta posición corresponde a jóvenes de los estratos socio-económicos bajo y medio-bajo, aunque la proporción de casos sólo supera levemente el $10 \%$ de este segmento. Un $49 \%$ de los encuestados indica que su proyecto personal considera terminar una carrera universitaria (tendencia que se eleva al $90 \%$ de los casos de los estudiantes del nivel socio-económico alto), mientras que un $22 \%$ de la muestra piensa que lo hará en una carrera técnica de nivel superior (siendo predominante el porcentaje de casos que señala esta alternativa en el estrato socio-económico bajo y medio-bajo). Un 4,5\% de los encuestados señala que optará por una carrera militar o policial, mientras que sólo un $7 \%$ manifiesta no tener aun claro cuál será su situación personal en el futuro. Esta información se sintetiza en la siguiente tabla: 
Estudios Pedagógicos, vol. XL, n. 1, 243-261, 2014

¿LAS COSAS CLARAS? ASPIRACIONES DE FUTURO Y PROYECTO EDUCATIVO LABORAL DE JÓVENES ESTUDIANTES SECUNDARIOS

Tabla 1. Aspiraciones de futuro personal en \% por nivel socio-económico del Establecimiento educativo

\begin{tabular}{|c|c|c|c|c|c|c|}
\hline & Total & $\begin{array}{c}\text { NSE } \\
\text { Bajo }\end{array}$ & $\begin{array}{c}\text { NSE } \\
\text { Medio } \\
\text { bajo }\end{array}$ & $\begin{array}{c}\text { NSE } \\
\text { Medio }\end{array}$ & $\begin{array}{c}\text { NSE } \\
\text { Medio } \\
\text { alto }\end{array}$ & $\begin{array}{c}\text { NSE } \\
\text { Alto }\end{array}$ \\
\hline Trabajar y no va a estudiar & 7,7 & 13.5 & 12.2 & 5.8 & 2.8 & 1.4 \\
\hline Terminará una carrera en la Universidad & 48,8 & 30.7 & 28.8 & 46.1 & 68.8 & 90.1 \\
\hline Terminará estudios en un IP o CFT & 22,1 & 35.0 & 30.2 & 22.2 & 14.0 & 2.8 \\
\hline Terminará una carrera Militar o Policial & 4,5 & 6.7 & 4.5 & 4.7 & 5.1 & 1.9 \\
\hline Cuidará a la familia y no va a estudiar & 0,3 & 0.6 & 0.3 & 0 & 0.6 & 0 \\
\hline No lo tiene claro & 7,1 & 7.4 & 9.3 & 7.3 & 5.3 & 3.3 \\
\hline Otro & 9,5 & 6.1 & 14.7 & 14.0 & 3.4 & 0.5 \\
\hline Total & 100 & 100 & 100 & 100 & 100 & 100 \\
\hline
\end{tabular}

La opción de no continuar con los estudios al egreso de la enseñanza media es señalada por el $11 \%$ de quienes se han formado bajo la modalidad EMTP y sólo un $2.7 \%$ de quienes lo hacen bajo el sistema EMCH. La alta proporción de casos de estudiantes de la formación técnico profesional que en sus aspiraciones privilegian la continuidad de estudios, ratifica la constatación de investigaciones recientes que señalan que, para la gran mayoría de los estudiantes de esta modalidad, no se trata de un modelo formativo terminal y de ingreso temprano al mundo del trabajo, como pudo haber ocurrido hace algunas décadas atrás (Sepúlveda et al., 2010; Sevilla, 2011).

En el marco de esta tendencia general, sin embargo, las aspiraciones o proyectos de futuro tienen una expresión temporal diferenciada de acuerdo a las características de los propios estudiantes. En efecto, cuando se les pregunta qué piensan hacer al año siguiente del egreso de $4^{\circ}$ medio, el porcentaje de casos que señala que se dedicará solo a trabajar se eleva al $21 \%$ del total de los encuestados. Este incremento se verifica, principalmente, en los estratos socio-económicos bajo y medio-bajo, y fundamentalmente entre quienes estudian la modalidad técnico profesional:

Tabla 2. Expectativas de futuro inmediato en \% por nivel socio-económico del establecimiento educativo

\begin{tabular}{|c|c|c|c|c|c|c|}
\hline & Total & $\begin{array}{c}\text { NSE } \\
\text { Bajo }\end{array}$ & $\begin{array}{c}\text { NSE } \\
\text { Medio } \\
\text { bajo }\end{array}$ & $\begin{array}{c}\text { NSE } \\
\text { Medio }\end{array}$ & $\begin{array}{c}\text { NSE } \\
\text { Medio } \\
\text { alto }\end{array}$ & $\begin{array}{c}\text { NSE } \\
\text { Alto }\end{array}$ \\
\hline Trabajar & 21,2 & 30.9 & 37.6 & 15.8 & 6.8 & 1.4 \\
\hline Ingreso a Educación Superior $^{\text {Carrera militar/policial }}{ }^{6}$ & 57,8 & 49.3 & 42.2 & 56 & 73.2 & 84.1 \\
\hline Carrat & 4,5 & 6.2 & 4.9 & 5.9 & 3.7 & 1.4 \\
\hline
\end{tabular}

5 La categoría Otro reúne las respuestas alternativas que definen los propios estudiantes. En este caso, la mayoría se manifiesta por una combinación de actividades de estudio y trabajo que no calzan, necesariamente, con las alternativas propuestas en el cuestionario, aunque, en un sentido estricto responden mayormente a opciones de estudio y postergación del ingreso al mundo del trabajo. Con todo, para fines del análisis se optó mantenerla como una categoría aparte.

$6 \quad$ Incluye la opción de realización del servicio militar al egreso de la enseñanza media 


\begin{tabular}{|c|c|c|c|c|c|c|}
\hline Preuniversitario & 4,5 & 1.3 & 2.5 & 4.4 & 9.6 & 3.7 \\
\hline No hará nada & 0,5 & 0 & 0.7 & 0.6 & 0.3 & 0.9 \\
\hline No lo tiene claro & 6,1 & 6.8 & 6.1 & 7.3 & 3.7 & 4.3 \\
\hline Otro & 5,4 & 5.5 & 5.9 & 10.0 & 2.7 & 4.2 \\
\hline Total & 100 & 100 & 100 & 100 & 100 & 100 \\
\hline
\end{tabular}

Un poco más de la mitad de los jóvenes manifiesta la intención de ingresar a la educación superior inmediatamente después de finalizada su enseñanza media, privilegiando un modelo de trayectoria lineal en la continuidad de sus estudios. Esta perspectiva se vuelve predominante entre aquellos estudiantes que provienen de los sectores socio-económicos medio-alto y alto. A diferencia de lo anterior, un porcentaje relevante de casos de los jóvenes de los estratos medio-bajo y bajo (cerca de un tercio de los encuestados de estos segmentos) evidencia en sus respuestas una perspectiva de trayectoria no lineal, que incluye el desarrollo de una actividad laboral en combinación con los futuros estudios. La incertidumbre de ingreso a un centro de educación superior y, muy particularmente, la imposibilidad de financiamiento de una carrera profesional en lo inmediato, son los principales factores que concurren en esta situación.

La noción de largo plazo en el cumplimiento de sus aspiraciones, se expresa de manera patente en el discurso de los jóvenes de nivel socio-económico bajo participantes de los grupos de discusión; así lo refleja una estudiante de un centro de educación media técnico profesional:

"Ahora estoy estudiando secretariado, pero la diferencia es que yo no quiero seguir siendo secretaria, sino que quiero estudiar pedagogía diferencial. Esto me sirve, porque ahí yo tengo algo seguro y estable; pero para poder estudiar igual encuentro que me va a costar, porque recursos para estudiar no tengo... por eso pretendo trabajar un año y después estudiar la carrera que me interesa".

Esta perspectiva se ve reforzada con las respuestas que se obtienen acerca de la disposición de combinar estudios y trabajo. Del total de encuestados que aspira a realizar estudios superiores inmediatamente después de su egreso de la enseñanza media, un 60,3\% manifiesta su disposición a realizar algún tipo de actividad laboral en algún momento del año. Aunque la modalidad de combinación de trabajo y estudios es muy variable (durante las vacaciones, los fines de semanas o algunas horas en la semana), ${ }^{7}$ lo importante de destacar aquí son las diferencias observadas por la disposición a hacerlo en función del nivel socio-económico de los estudiantes. Como puede verse en el gráfico siguiente, mientras que un porcentaje cercano al $80 \%$ de los estudiantes de los estratos socio-económicos medios y bajos señalan su disposición a combinar experiencias de trabajo y estudio en este período, esta tendencia decrece al $50 \%$ en el estrato medio alto y en el caso del estrato socio-económico alto, el porcentaje de casos que manifiesta esta disposición sólo supera levemente el $20 \%$.

En los grupos de discusión se profundizó en este aspecto, observándose una cierta desinformación de los jóvenes acerca de las posibilidades reales de lograr este objetivo. El discurso, en este sentido, responde mayormente a una declaración de intenciones, sin que se observen planes o perspectivas claras de orientación hacia una actividad laboral. Mayoritariamente las referencias son a actividades laborales acotadas en período de vacaciones, de baja exigencia de habilidades previas. En el caso de los estudiantes de la modalidad EMTP, las referencias son, por lo general, a espacios vinculados a su práctica laboral durante el período formativo o la búsqueda de alternativas a partir del contacto o referencia de sus profesores o directivos del lugar donde estudiaron. 
Gráfico 1. Disposición a combinar estudio y trabajo; casos estudiantes que pretenden estudiar al egreso de enseñanza media, en \% por nivel socio-económico del establecimiento educativo

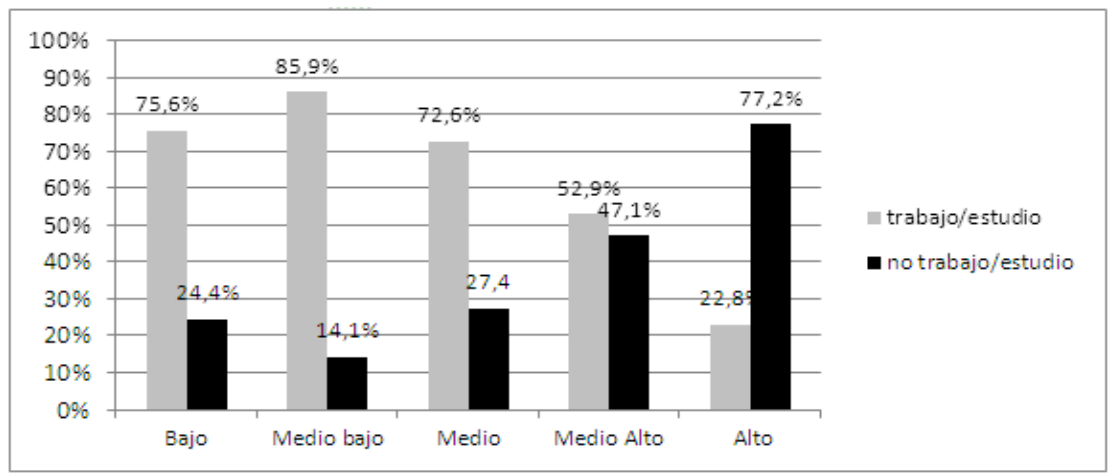

De este modo, aunque los jóvenes estudiantes comparten aspiraciones de continuidad de estudios superiores, en la definición de sus proyectos personales se hacen evidentes las diferencias de tipo socio-económicas en la consecución de sus objetivos. Como consecuencia, la necesidad de emprender una actividad laboral previo a la realización de estudios o la combinación de estudios y trabajo en un período prolongado de tiempo, es una alternativa o necesidad cierta para una gran mayoría de los jóvenes de menores recursos, cuestión que incide en una proyección de una trayectoria educativa más prolongada y menos lineal en comparación a los estudiantes de estratos socio-económicos superiores.

Por cierto, esta situación puede ser sorteada por aquellos estudiantes que alcanzan un buen rendimiento académico, lo que les permitiría acceder a becas para el financiamiento de sus estudios; en este contexto parece pertinente preguntar acerca de la autopercepción de capacidades personales, del grado de certeza que tienen los estudiantes sobre sus futuros resultados de la prueba de selección universitaria (PSU), las expectativas sobre alternativas académicas a su alcance y la consistencia de estas con las aspiraciones ya reseñadas. En los acápites siguientes se revisan estas dimensiones recogidas a lo largo de la investigación.

4.2. AUTO-EVALUACIÓN DE COMPETENCIAS ESCOLARES Y EXPECTATIVAS DE LOGROS ACADÉMICOS EN LA SELECCIÓN UNIVERSITARIA

Situados ante una autoevaluación del manejo de competencias fundamentales de la vida escolar, los jóvenes estudiantes tienen una visión positiva de su propio desempeño. Casi el 50\% de los encuestados se sitúa en los niveles más altos de una escala de autoevaluación referida a capacidad de análisis y un $65 \%$ lo hace respecto a un nivel de manejo adecuado de comprensión lectora. ${ }^{8}$ La capacidad de resolución de problemas prácticos, el saber expresarse en público y, muy particularmente, el uso de las tecnologías de comunicación, concentra respuestas de buen nivel de desempeño en porcentajes cercanos al $60 \%$ de los encuestados, sin que se observen diferencias importantes por modalidad de estudios ni por nivel socio-económico de los mismos.

8 La forma de auto-calificación en la encuesta consistió en la definición de una escala continua de 1 a 5 , solicitándoles a los estudiantes que se ubicaran en algunos de los puntos respecto a cada área temática abordada. 
El único ámbito que presenta una menor recurrencia de valoraciones positivas en el proceso auto-evaluativo es el de conocimiento matemático, pero sólo un tercio de los encuestados se sitúa en el polo de respuestas que reconoce deficiencias o limitaciones en este ámbito. De igual manera, la mayoría de los estudiantes tiene una valoración favorable del espacio escolar de su establecimiento y una buena evaluación de los docentes y su apoyo pedagógico, aspecto que se ve reforzado por una auto-percepción positiva de su propio desempeño y capacidades escolares al finalizar su enseñanza media. Lo anterior, sin embargo, contrasta con las expectativas de los resultados que alcanzarán al rendir la prueba de selección universitaria, evidenciándose, para un segmento importante de los estudiantes, una disociación entre la auto-evaluación de sus resultados escolares y el pronóstico de logro en esta instancia.

El 75\% de los jóvenes participantes de este estudio señaló que rendiría la PSU en el año de su egreso de $4^{\circ}$ medio. Entre estos, manifestaban su intención de presentarse al examen el $96 \%$ de quienes estudiaron bajo la modalidad científico humanista, y el $74.6 \%$ de los estudiantes que cursaban sus últimos años de enseñanza media bajo la modalidad EMTP. Los estudiantes del estrato socio-económico medio-bajo son los que presentan el mayor porcentaje de casos que no rendiría la PSU (33\%), lo que contrasta con aquellos del nivel alto, donde no la rendiría sólo el $0.5 \%$ de este grupo.

Ahora bien, al consultarse acerca del puntaje que pensaban obtener en este examen de admisión a la educación superior, el 43,2\% de los encuestados manifestó que este sería de 600 o más puntos, existiendo un $56,8 \%$ del total que cree que su puntaje sería inferior a los 600 puntos ponderados. Las expectativas de logro académico analizado por el nivel socio-económico de los jóvenes establece una fuerte diferenciación por estratos: mientras que sólo $13,1 \%$ de los jóvenes del nivel socio-económico bajo tenía expectativas de superar la meta de los 600 puntos, esta alcanzaba al 87,4\% de quienes pertenecían al estrato socio-económico alto. Los resultados se resumen en la siguiente tabla:

Tabla 3. Expectativas de puntaje a lograr en PSU, en \% por nivel socio-económico del establecimiento educativo

\begin{tabular}{|c|c|c|c|c|c|c|}
\hline & Total & $\begin{array}{c}\text { NSE } \\
\text { Bajo }\end{array}$ & $\begin{array}{c}\text { NSE } \\
\text { Medio } \\
\text { bajo }\end{array}$ & $\begin{array}{c}\text { NSE } \\
\text { Medio }\end{array}$ & $\begin{array}{c}\text { NSE } \\
\text { Medio } \\
\text { alto }\end{array}$ & $\begin{array}{c}\text { NSE } \\
\text { Alto }\end{array}$ \\
\hline Menos de 449 puntos & 8,6 & 23.7 & 13.6 & 6.7 & 2.8 & 0.5 \\
\hline Entre 450 y 549 puntos & 24,3 & 35.1 & 33.8 & 28.9 & 17.7 & 3.9 \\
\hline Entre 550 y 599 puntos & 23,9 & 25.2 & 27.1 & 29.9 & 24.2 & 8.3 \\
\hline Entre 600 y 649 puntos & 16,7 & 8.4 & 17.0 & 20.1 & 19.0 & 13.2 \\
\hline Entre 650 y 699 puntos & 13,4 & 3.1 & 4.8 & 10.9 & 19.6 & 29.3 \\
\hline Entre 700 y 749 puntos & 8,4 & 0.8 & 1.6 & 2.5 & 11.0 & 29.8 \\
\hline 750 puntos o más & 4,7 & 0.8 & 2.1 & 1.1 & 5.8 & 15.1 \\
\hline Total & 100 & 100 & 100 & 100 & 100 & 100 \\
\hline
\end{tabular}

Las proyecciones de los resultados de la prueba de selección universitaria a partir de la auto calificación personal en esta encuesta, presenta similitudes y diferencias con el comportamiento real de los resultados en los últimos años medido por estrato socioeconómico; por una parte, es bastante ajustado para el caso de los jóvenes de estratos 
socio-económicos bajo y medio-bajo, existiendo como contrapartida, un exceso de optimismo en el caso de los jóvenes de nivel socio-económico medio alto y alto. En este último caso, el porcentaje de estudiantes que proyecta un puntaje de 600 puntos o más en el examen de admisión universitaria, supera en más de 30 puntos las tendencias observadas recientemente en los resultados de la PSU (Contreras et al., 2007).

Muy relacionado a lo anterior, la percepción acerca del grado de preparación para iniciar una vida de estudiantes luego del egreso de la enseñanza media, evidencia una diferencia de tipo socio-económica relevante. Son los jóvenes de los estratos superiores y, muy particularmente, los de nivel alto, quienes consideran que su preparación es adecuada para hacer frente a la prueba de selección universitaria y el desempeño en una carrera de estudios superiores. La percepción de estar adecuadamente preparado para la rendición de la PSU aumenta en un poco más de 20 puntos en este grupo en comparación con los estudiantes de nivel socio-económico bajo, tal como se expresa en el siguiente gráfico:

Gráfico 2. Percepción sobre el nivel de preparación para alcanzar un buen rendimiento en la PSU, por nivel socio-económico del establecimiento educativo

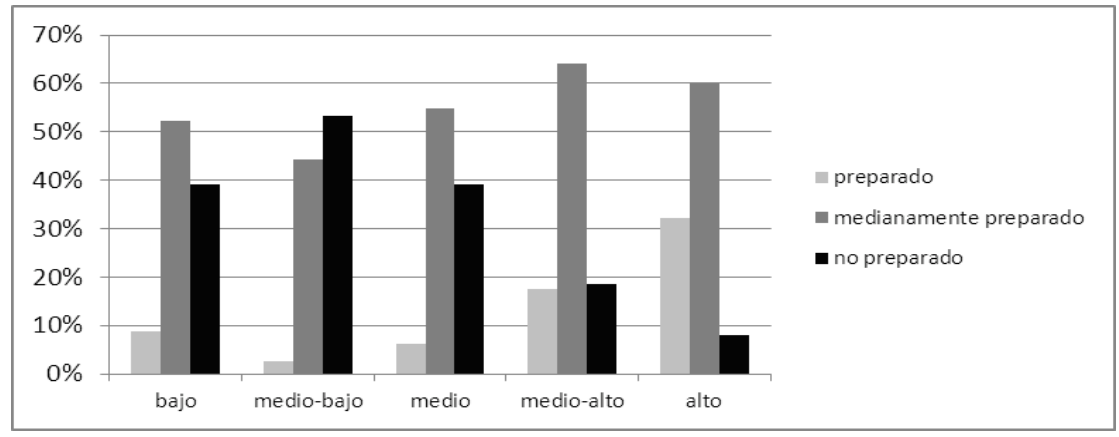

Los testimonios recogidos en los grupos de discusión, evidencian experiencias disímiles en la preparación de los jóvenes estudiantes durante los últimos años de su formación en la enseñanza media. Mientras que los estudiantes de establecimientos de nivel socio-económico alto, mayoritariamente señalan que han desarrollado una estrategia de reforzamiento formativo orientada por el propio establecimiento donde cursan su enseñanza, en el caso de los jóvenes de nivel socio-económico bajo, las experiencias son variables, siendo más recurrente la constatación de ausencia de presiones institucionales, y el hecho de que, tardíamente, los jóvenes se han dado cuenta de la importancia de prepararse para esta examinación.

De un modo más general, es posible sostener que en uno y otro caso, la evaluación del esfuerzo educativo varía en relación a la proyección personal de los estudiantes y la consideración del valor atribuido a la educación recibida. En no pocos casos de jóvenes pobres es posible observar una orientación instrumental frente a los estudios y un cuestionamiento de la utilidad de los mismos. ${ }^{9}$ Desde este punto de vista es posible interpretar las causas del desajuste observado entre la auto percepción positiva del manejo de competencias escolares en la enseñanza media y la proyección de resultados

9 Véase las referencias a la experiencia escolar de estudiantes en el sistema educativo francés de Dubet y Martuccelli y la referencia a los distintos modelos de estudiantes y sus proyectos educativos (1998: 342). 
negativos que prevén al rendir el examen de admisión al sistema universitario. Las dos citas siguientes sirven de contraste para el análisis de la experiencia de los jóvenes en su proceso educativo al finalizar la enseñanza media: ${ }^{10}$

“...yo comencé a pensar y elegir que quería estudiar en $2^{\circ}$ medio, cuando tenía que elegir un electivo, porque ahí había que empezar a preparar la PSU para lo que quería estudiar. Los profes te orientan de acuerdo al mismo rendimiento que uno tiene, aunque uno elige de acuerdo a lo que quiere y como evalúa su rendimiento en las pruebas que se hacen; me incliné por las matemáticas y eso me llevó a buscar alternativas en el área de la ingeniería..."

"uno siempre estudia solo para una prueba; en la casa o los profes te dicen 'trata de estudiar aunque sea una hora al día por lo menos' pero uno nunca lo hace, porque el colegio es... yo personalmente creo que el colegio es como un puente que hay que cruzar para poder conseguir lo que uno quiere; como que el colegio es como a lo que uno viene a pasarlo no más; a mi me va bien en el colegio, pero nunca me he preocupado de estudiar más allá del momento antes de la prueba...mis expectativas yo creo que las voy a cumplir, no creo que me vaya muy bien en la PSU, pero igual voy a poder estudiar después..."

De este modo, aunque de manera transversal, los estudiantes valorizan su experiencia educativa, dándola por buena o satisfactoria, de cara a una instancia de evaluación externa como la PSU, prevalece un criterio de realismo que establece los límites entre lo factible y lo deseable, evidenciando las diferencias de nivel formativo alcanzado y las posibilidades de proyección académica, altamente diferenciadas por el tipo de centros educacionales donde estos jóvenes estudiaron.

Lo anterior, sin embargo, no es un obstáculo para la existencia de una disposición positiva hacia el futuro y la emergencia de un discurso que enfatiza en la confianza por alcanzar las aspiraciones individuales; de hecho, el porcentaje de casos que señala estar confiado en lograr sus aspiraciones de ingreso a un centro de educación superior es levemente mayor en los jóvenes de estratos bajos en comparación a los de nivel superior, pese a reconocer que sus puntajes de PSU serán deficitarios. Como se verá, esta situación responde mayormente a un ejercicio de ajuste los estudiantes en relación a una estructura diferenciada de oportunidades presente en el sistema de educación superior de nuestro país, aspecto que revela la existencia de modelos de ambición o aspiración individual segmentados por el nivel socio-económico.

\subsection{CRITERIOS DE ELECCIÓN DE ESTUDIOS SUPERIORES: AJUSTES DE PROYECTOS PERSONALES A PARTIR DE LA OFERTA DISPONIBLE}

Los estudiantes que manifiestan su disposición a continuar estudios inmediatamente después de finalizado su $4^{\circ}$ año de enseñanza media, en un porcentaje importante, señalan tener claridad sobre sus intereses educativos: el $63 \%$ de los encuestados manifiesta tener completamente clara su decisión sobre el área formativa de interés, un $25 \%$ señala no haber decidido su opción, mientras que sólo un $4 \%$ manifiesta abiertamente no tener claridad sobre la alternativa de estudios superiores. En la elección de una carrera específica

10 Se trata de dos hombres, uno que estudia en un establecimiento particular pagado y otro, perteneciente a un establecimiento de dependencia particular subvencionado de nivel socio-económico medio-bajo. Por cierto, las citas representan dos modelos discursivos predominantes entre los jóvenes, sin que lo anterior signifique que no existan otras orientaciones subjetivas respecto a este tema. 
aumenta el porcentaje de casos que señala no haberse decidido totalmente, manteniéndose el porcentaje marginal que declara no tener una opción. La definición del lugar de estudio de interés es la que presenta una mayor indefinición entre los encuestados, aunque el porcentaje de casos que señala no tener ninguna claridad sólo llega al 13\% del total.

La consideración de estas respuestas en función del nivel socio-económico muestra similitudes, salvo en el caso de los jóvenes de estratos socio-económicos altos que presentan, particularmente, mayor claridad en su opción del centro de estudios donde desean cursar su educación superior:

Tabla 4. Grado de claridad sobre elección de estudios superiores en \% por nivel socio-económico del establecimiento educativo (\% de casos que señalan tener elección completamente clara)

\begin{tabular}{|c|c|c|c|c|c|}
\hline Dimensión & Bajo & Medio bajo & Medio & Medio alto & Alto \\
\hline $\begin{array}{c}\text { Área temática } \\
\text { de interés }\end{array}$ & 61.5 & 53.7 & 47.7 & 68.9 & 84.7 \\
\hline Carrera & 60.3 & 53.9 & 67.2 & 56.2 & 66.3 \\
\hline $\begin{array}{c}\text { Centro de } \\
\text { estudios }\end{array}$ & 37.3 & 35.5 & 42.8 & 51.6 & 57.7 \\
\hline Total & 100 & 100 & 100 & 100 & 100 \\
\hline
\end{tabular}

Esta tendencia en las respuestas evidenciaría un grado importante de reflexión para la decisión de futuro educativo de los jóvenes; la mayoría señala tener claridad y haber tomado una decisión sobre el área temática de su interés, aunque esta claridad decrece respecto a la carrera y el centro de estudios donde poder desarrollar su proceso formativo. En los grupos de discusión realizados en el marco del estudio fue posible observar una tendencia discursiva que marcaba la adaptación de las aspiraciones a alternativas factibles de realización (por ejemplo, la elección de una carrera en una misma área temática al reconocer la dificultad de ingreso a la opción inicial). Varios de los estudiantes participantes señalaron que en los años previos de su enseñanza media podían soñar con estudiar una determinada carrera pero que, en el último año de su formación, su opción debía ser más aterrizada y acorde a sus propias posibilidades de realización.

Entre los posibles obstáculos que pudiesen limitar el cumplimiento de los objetivos trazados, los jóvenes destacan con igual importancia el puntaje alcanzado en la PSU, el costo o arancel de la carrera y el promedio de notas alcanzado en la enseñanza media. Sin embargo, al analizar las respuestas por nivel socio-económico, se observan algunas diferencias importantes; los jóvenes pertenecientes al grupo socio-económico bajo atribuyen, proporcionalmente, una menor incidencia a las notas de la enseñanza media en la identificación de obstáculos (la diferencia con los estudiantes de nivel alto es de casi 20 puntos); en contraste, la diferencia existente por nivel socio-económico en la consideración del costo o arancel de la carrera como obstáculo para lograr estudiar lo que se desea es muy significativa, presentando una diferencia de casi 60 puntos entre los grupos bajo y alto. 
Tabla 5. Percepción de obstáculos que incidirían elección estudios superiores; porcentaje de casos menciones por nivel socio-económico del establecimiento educativo ${ }^{11}$

\begin{tabular}{|c|c|c|c|c|c|c|}
\hline & Total & $\begin{array}{c}\text { NSE } \\
\text { Bajo }\end{array}$ & $\begin{array}{c}\text { NSE } \\
\text { Medio } \\
\text { bajo }\end{array}$ & $\begin{array}{c}\text { NSE } \\
\text { Medio }\end{array}$ & $\begin{array}{c}\text { NSE } \\
\text { Medio } \\
\text { alto }\end{array}$ & $\begin{array}{c}\text { NSE } \\
\text { Alto }\end{array}$ \\
\hline Notas de la enseñanza media & 51.0 & 39.0 & 46.2 & 52.1 & 62.6 & 56.7 \\
\hline Puntaje alcanzado en PSU & 58.6 & 63.2 & 59.1 & 65.8 & 56.3 & 68.5 \\
\hline Costo o arancel de la carrera & 58.3 & 77.9 & 79.4 & 72.2 & 57.8 & 20.2 \\
\hline Oposición de los padres & 3.2 & 2.6 & 2.8 & 4.6 & 3.1 & 3.4 \\
\hline Otro & 4.4 & 3.9 & 5.6 & 6.2 & 3.4 & 4.0 \\
\hline
\end{tabular}

Colocados ante la alternativa de privilegiar una opción a la hora de iniciar estudios superiores, la mayoría de los estudiantes (63\% de las respuestas) privilegia la carrera de interés. Sólo un 13\% de los encuestados preferiría elegir el centro de estudios, aunque en el grupo socio-económico alto este porcentaje se eleva a casi el $21 \%$ de quienes responden la encuesta. Es posible que la selectividad y distinción por tipo de institución juegue un rol importante en la visión de los jóvenes pertenecientes a este grupo socio-económico.

Tabla 6. Factor que privilegia en elección estudios superiores; \% por nivel socio-económico del establecimiento educativo

\begin{tabular}{|c|c|c|c|c|c|c|}
\hline & Total & $\begin{array}{c}\text { NSE } \\
\text { Bajo }\end{array}$ & $\begin{array}{c}\text { NSE } \\
\text { Medio } \\
\text { bajo }\end{array}$ & $\begin{array}{c}\text { NSE } \\
\text { Medio }\end{array}$ & $\begin{array}{c}\text { NSE } \\
\text { Medio } \\
\text { alto }\end{array}$ & $\begin{array}{c}\text { NSE } \\
\text { Alto }\end{array}$ \\
\hline El centro de estudios de interés & 13,3 & 6.7 & 8.8 & 12.5 & 14.8 & 20.8 \\
\hline La carrera que le interesa & 63,3 & 69.3 & 69.6 & 59.9 & 59.2 & 62.3 \\
\hline El área temática que le interesa & 23,4 & 24.0 & 21.6 & 27.6 & 26.1 & 16.9 \\
\hline Total & 100 & 100 & 100 & 100 & 100 & 100 \\
\hline
\end{tabular}

La opción del centro de estudios donde cursar la enseñanza post-secundaria implica la consideración de un conjunto de factores que pesan en la determinación de los jóvenes y sus familias. La tabla siguiente intenta indagar sobre estas opciones en términos globales. Dos son las principales afirmaciones que aglutinan el máximo de opciones de los encuestados; la calidad de la formación impartida y el prestigio de la institución. Estas opciones, por cierto, remiten a un juicio general que demanda una oferta formativa funcional al esfuerzo de continuar estudios una vez finalizada la enseñanza media. Sin embargo, el prestigio institucional es un criterio presente de manera más recurrente en los jóvenes de nivel socio-económico medio-ato y alto en comparación con el resto, cuestión que también se observa en la mayor proporción de casos que señalan el interés o disposición de elegir una universidad perteneciente al Consejo de Rectores. A diferencia de lo anterior, el valor de los aranceles de las carreras constituye un factor de peso para un grupo

11 Pregunta de respuesta de opción múltiple. 
relevante de estudiantes de los sectores socio-económicos medio y bajos, reduciéndose considerablemente su consideración entre los jóvenes de nivel socio-económico superior.

Sobre los factores de elección de la carrera, en los grupos de discusión se destacó que muchos jóvenes desarrollan estrategias de indagación y análisis de las características y posibilidades que entregan las alternativas a las que se deseaba optar. Particularmente entre los estudiantes de los estratos medios y bajos se hizo mención a la búsqueda en la WEB de información institucional sobre la oferta existente, así como algunos sitios gubernamentales de orientación al postulante (como, por ejemplo, aquellos que informan sobre inserción laboral y promedio de ingresos de quienes se titulan en las respectivas especialidades); con todo, tales acciones muchas veces no tienen suficiente orientación, reconociéndose, en muchos casos, que los intereses están orientados por definiciones relativamente vagas. A diferencia de lo anterior, en los grupos de jóvenes de ingresos altos existe mayor referencia a la orientación de apoyo en el establecimiento educativo y la definición temprana de lo que podríamos definir como una orientación vocacional absoluta, esto es, la asociación del interés de estudios de una carrera en una universidad en particular, y la baja consideración de antecedentes sobre ingreso laboral y remuneraciones futuras en relación a sus respectivas opciones.

Lo anterior se ve corroborado cuando se plantea a los estudiantes la alternativa de ingreso a educación superior por centro de estudios; ${ }^{12}$ aunque del total de respuestas un poco más del $70 \%$ de los encuestados considera que las universidades tradicionales públicas tienen más prestigio que el resto, en la definición de opciones parecen primar razones diversas como justificativo de esta selección, predominando la posibilidad de ingreso por puntaje alcanzado en PSU y la accesibilidad al financiamiento de la carrera.

En el caso de los estudiantes de estratos socio-económicos más pobres, existe una mayor apertura a la selección de centros de educación superior de tipo no selectivo. A diferencia de lo anterior, en los grupos socio-económico alto y medio-alto predomina, sin restricciones, la elección de una universidad selectiva. El gráfico siguiente sintetiza las respuestas a partir de la selección de centros de educación superior por parte de los estudiantes encuestados:

Gráfico 3. Opción de centros de estudios para cursar estudios superiores en \% por nivel socio-económico por nivel socio-económico del establecimiento educativo

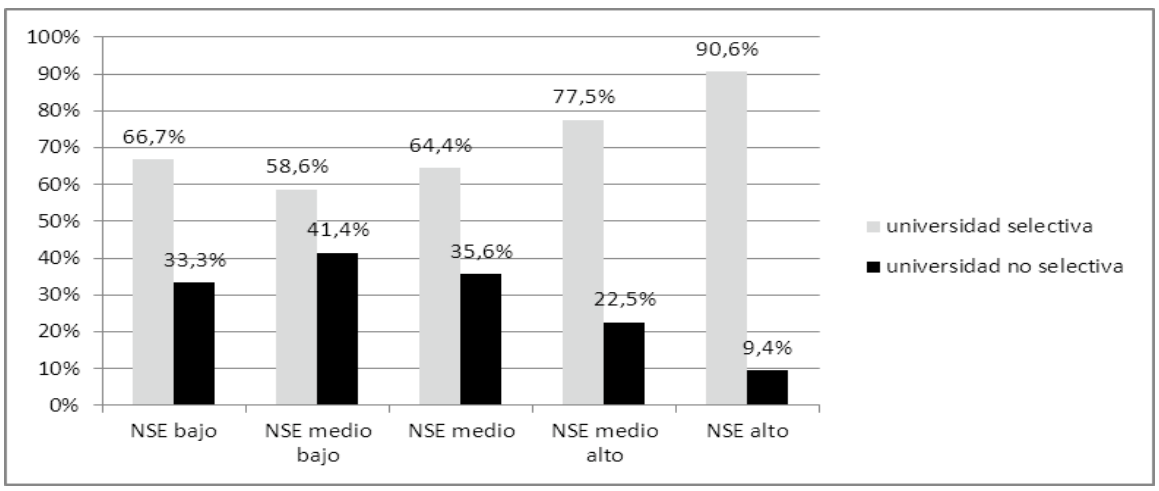

12 En la encuesta se entregó un listado con alternativas para la selección individual de los estudiantes. La agrupación que se realiza en el gráfico siguiente se organiza a partir de la clasificación de centros de educación superior que ofrece el estudio de Torres y Zenteno (2011). 
Tratándose de un ejercicio libre de expresión de un deseo o interés (esto es, la especificación de las aspiraciones planteadas por los estudiantes), los datos presentados en este artículo permiten sostener que en las respuestas se evidencian diferencias de estrategias acorde a la realidad educativa y socio-económica de los sujetos. Entre los jóvenes de nivel socio-económico bajo y medio bajo, se reconoce las dificultades de lograr buenos resultados en la prueba de selección a la educación superior; a partir de esto, un segmento importante, en sus estrategias personales manifiesta la apertura a alternativas para el cumplimiento de sus aspiraciones, lo que incluye la elección de centros educativos con menores exigencias en sus requisitos de ingreso. En contraste, los jóvenes de los estratos superiores, aunque confían en lograr buenos resultados en la PSU, tienen altas aspiraciones de estudiar en universidades selectivas y de mayor prestigio, incorporando con esto una cuota de incertidumbre, aunque distinta, igualmente relevante sobre su destino final en la educación superior.

\section{CONCLUSIONES}

Los resultados que arroja este estudio permiten sostener, en primer lugar, que la gran mayoría de los jóvenes, independientemente del establecimiento donde estudiaron o el nivel socio-económico al que pertenecen, aspiran a cursar estudios superiores una vez finalizada su enseñanza secundaria. El cierre del ciclo formativo y el ingreso temprano al mundo del trabajo, es una decisión que atañe solo a un grupo marginal de la muestra, aunque fuertemente concentrada en el segmento de familias de menores ingresos.

Esta tendencia masiva a privilegiar la continuidad de estudios, sin embargo, presenta diferencias importantes cuando se analizan las expectativas temporales de realización y las opciones efectivas de ingreso a la educación superior y la eventual articulación con actividades laborales que son señaladas por los estudiantes. En este ámbito es posible distinguir una diferenciación de carácter socio-económico y la emergencia de diversas racionalidades que darían cuenta de un ajuste de las aspiraciones de futuro y los itinerarios posibles de alcanzarlos entre los jóvenes estudiantes. El peso de los requerimientos económicos para la continuidad de estudios y la incertidumbre frente a las exigencias académicas de ingreso a la educación superior, son dos factores relevantes que inciden mayormente en las orientaciones de los estudiantes de menores recursos.

Aunque se observa un alto nivel de aspiraciones en el conjunto de los jóvenes, la elección previa de centros de educación superior en función al origen social y el capital socio-cultural disponible, evidenciaría una tendencia a la reproducción de la segmentación propia de los establecimientos educacionales donde estos estudiaron, y que resulta funcional al modelo de segmentación sistémica que se observa en la estructura de los centros de educación superior en nuestro país. Un grupo relevante de los jóvenes de nivel socio-económico bajo y medio-bajo, reconociendo las dificultades de logro académico medido en la prueba de selección universitaria, menos que manifestar desafección hacia la alternativa de estudios post-secundarios, visualizan en opciones académicas menos exigentes (universidades no selectivas) una alternativa para el cumplimiento de sus aspiraciones. En el caso de los jóvenes de nivel socio-económico alto, la incertidumbre es también un rasgo destacable, toda vez que las ambiciones de alcanzar un tipo de carrera profesional y estudiar en un centro de estudios distintivo, constituye un anhelo ampliamente generalizado. 
Aunque hermanados generacionalmente en la construcción de aspiraciones de futuro ambiciosas, las trayectorias posibles que construyen los jóvenes de manera previa parecen orientarse hacia caminos distintos, condicionados por el nivel socio-económico de origen y el establecimiento educacional donde estos estudiaron. La existencia de una oferta de educación superior segmentada social y económicamente, tal como se ha demostrado en estudios recientes (Brunner y Uribe, 2007; Orellana, 2011), parece ser un canal que, sin limitar el anhelo de superación y el alto grado de ambición presente en esta generación, puede eventualmente resolver esta demanda, sin modificar mayormente las diferencias de origen y segmentación social prevalecientes en nuestra sociedad.

\section{REFERENCIAS BIBLIOGRÁFICAS}

Appadurai, A. (2004). The capacity to aspire: Culture and the terms of recognition. En Rao, V. y Walton, M. (Eds.), Culture and Public Action (pp. 58-84) California: Stanford University Press.

Berzin, S. (2010). Educational aspirations among low-income youths: examining multiple conceptual models. Children \& Schools, vol. 32, n.2, 112-124.

Brunner, J.J. y Uribe, D. (2007). Mercados universitarios. El nuevo escenario de la educación superior. Santiago: Ed. Universidad Diego Portales

Castillo, J. y Cabezas, G. (2010). Caracterización de jóvenes primera generación en educación superior. Nuevas trayectorias hacia la equidad educativa. Calidad en la Educación, n.32, 44-76.

Contreras, M., Corbalán, F. y Redondo, J. (2007). Cuando la suerte está echada: estudio cuantitativo de los factores asociados al rendimiento en la PSU. Revista Electrónica Iberoamericana sobre Calidad, Eficacia y Cambio en Educación vol.5, n.5e, 259-263. Consultado el 29.10 .2012 http://www.rinace.net/arts/vol5num5e/art35.pdf.

Donoso, S., Donoso, G., y Arias, O. (2010). Iniciativas de retención de estudiantes en educación superior. Calidad en la Educación, n.33, 15-61.

Dubet, F. y Martuccelli. D. (1998). En la Escuela: Sociología de la experiencia escolar. Buenos Aires: Losada.

Gaete, M. y Morales, R. (2011). Articulación del sistema de educación superior en Chile: posibilidades, tensiones y desafíos. Calidad en la Educación, n.35, 51-89.

Grytnes, R. (2011). Making the right choice! Inquiries into the reasoning behind young people's decisions about education. Young, vol.19, n.3, 333-351.

Jones, N. y Schneider, B. (2009). The influence of aspirations on educational and occupational outcomes. En Furlong, A. (Ed.), Handbook of youth and young adulthood: New perspectives and agendas (pp. 392-398) London: Routledge.

Lehmann, W. (2009). University as vocational education: working-class students' expectations for university. British Journal of Sociology of Education, vol. 30, n.2, 137-149.

(2004). 'For some reason, I get a little scared': structure, agency, and risk in school-work transitions. Journal of Youth Studies, Vol. 7, n.4, 379-396.

McDonough, P. (1997). Choosing Colleges; How social class and schools structure opportunity. New York: State University of New York Press.

Meller, P. (2010). Carreras Universitarias: rentabilidad, selectividad y discriminación. Santiago: Uqbar, Ediciones y Centro de Investigación Avanzada en Educación (CIADE), Universidad de Chile.

Meo, A. y Dabenigno, V. (2010). Expansión de las aspiraciones educativas en jóvenes de sectores populares. ¿Evidencias de la emergencia de un nuevo habitus escolar en la ciudad de Buenos Aires? Revista Iberoamericana de Educación, vol. 53, n.5, 1-13. 
Orellana, V. (2011). Nuevos estudiantes y tendencias emergentes en la Educación Superior: una mirada al Chile de mañana. En Jiménez, M. y Lagos, F. (Eds.), Nueva geografía de la educación superior y de los estudiantes: Una cartografía del sistema chileno, su actual alumnado y sus principales tendencias (pp. 80-142). Santiago: Ediciones Universidad San Sebastián.

Schneider, B. y Stevenson, D. (1999). The ambitious generation: America's teenager, motivated but directionless. New Haven: Yale University Press.

Sellar, S., Gale, T., y Parker, S. (2011). Appreciating aspirations in Australian higher education. Cambridge Journal of Education, Vol. 41, n.1, 37-52.

Sepúlveda, L., Ugalde, P., y Campos, F. (2010). La enseñanza media técnico profesional en Chile: orientaciones actuales desde la perspectiva de sus actores en Centro de Estudios MINEDUC, Evidencias para políticas públicas en Educación: Selección de Investigaciones Tercer Concurso FONIDE. Tomo I. (pp. 15-52). Santiago: Eds. MINEDUC.

Sevilla, P. (2001). Trayectorias laborales y educacionales de los egresados de enseñanza media técnico-profesional en Chile: Un análisis de la cohorte 2003. Serie Tendencias y Debates n6, RedEtis. Buenos Aires: IIPE-UNESCO.

Torres, R. y Zenteno, M.L. (2011). El sistema de educación superior: Una mirada desde las instituciones y sus características en Jiménez, M. y Lagos, F. (Ed.), Nueva geografía de la educación superior y de los estudiantes: Una cartografía del sistema chileno, su actual alumnado y sus principales tendencias (pp. 13-78) Santiago: Ediciones Universidad San Sebastián. 
